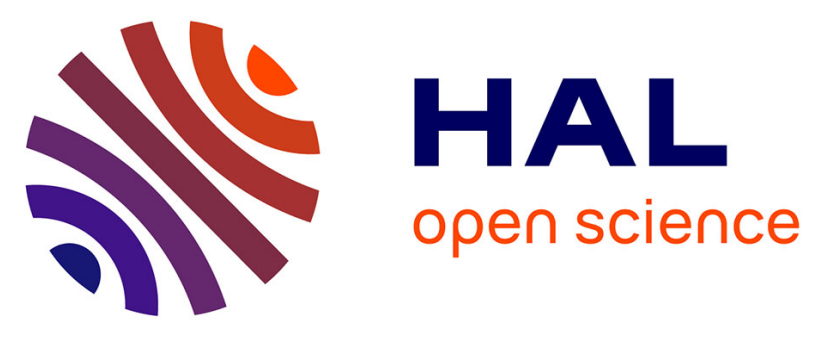

\title{
Models as Representations for Supporting the Development of e-Procedures
}

\author{
Marco Winckler, Philippe Palanque
}

\section{To cite this version:}

Marco Winckler, Philippe Palanque. Models as Representations for Supporting the Development of e-Procedures. Usability in Government Systems: User Experience Design for Citizens and Public Servants, 3 (1: Chapter 19: Models as Abstract Representations for supporting the development of e-Procedures), MDPI AG, Switzerland, pp.1-3, 2012, 978-0-12-391063-9. 10.3390/admsci3010001 . hal-02911555

\section{HAL Id: hal-02911555 \\ https://hal.science/hal-02911555}

Submitted on 3 Aug 2020

HAL is a multi-disciplinary open access archive for the deposit and dissemination of scientific research documents, whether they are published or not. The documents may come from teaching and research institutions in France or abroad, or from public or private research centers.
L'archive ouverte pluridisciplinaire HAL, est destinée au dépôt et à la diffusion de documents scientifiques de niveau recherche, publiés ou non, émanant des établissements d'enseignement et de recherche français ou étrangers, des laboratoires publics ou privés. 
Chapter 19 Models as Abstract Representations for supporting the development of eProcedures

\section{[NON PRINT ITEMS]}

Abstract: The development of successful electronic procedures is a complex activity that requires diverse expertise on administrative processes and software development. Such expertise requires a multidisciplinary team that should combine technical issues and expectations of all users, including citizens and administrative clerks. This chapter is aimed to help you to understand how model-based approaches can be used to deal with development of complex e-procedures and, in particular, to communicate design options to a multidisciplinary team. To this very purpose this chapter illustrates two kinds of models: tasks models that describe the actions user should perform at every step of an administrative procedure; and system models that describe how design options can be implemented by the system to support user tasks. When used altogether, these models provide useful information to understand the implications of individual user's tasks and the system's behavior along the different steps in the underling workflow of an e-procedure.

Key words: development process, model-based approach, e-procurement systems specification, task models.

\section{Author Contact Information:}

Marco Winckler

Assistant Professor of Computer Science

ICS-IRIT, Université Paul Sabatier, Toulouse, France

winckler@irit.fr

Telephone: +33 5.61.55.63.59

Philippe Palanque 
Professor of Computer Science

ICS-IRIT, Université Paul Sabatier, Toulouse, France

palanque@irit.fr

Telephone: $+33(0) 5.61 .55 .69 .65$

\section{[Chapter Starts here]}

[ChNum]Chapter 19

[ChTitle] Models as Abstract Representations for supporting the development of e-

Procedures

[H1]Introduction

Government agencies are increasingly moving towards providing web support for their administrative procedures. Such web applications must do five important things:

1. ensure the security of information exchange (e.g., authentication of users, date and time, secure transfer of data, etc. as discussed in the Chapter 14 [User issues in security])

2. provide an efficient notification system that helps all users (citizens and organizations) to monitor the progress of the process

3. provide flexible support to complex business rules (which might change according to new regulations and laws)

4. support data exchange among several databases and legacy systems

5. be accessible and usable for a large and diverse public of users.

The development of successful electronic procedures is a complex activity that requires diverse expertise in administrative processes and software development. Such expertise can only be acquired by a multidisciplinary team that should be able to harmoniously combine technical issues and expectations of all users, including 
citizens and administrative clerks (which are respectively called external and internal users [see this book outline]). Despite the fact that e-procurement applications often focus on citizens as target users, we should not forget the important role played by stakeholders. By "stakeholder" we mean administrative clerks and agents in charge of receiving citizens' applications, analyzing the data provided and deciding the outcomes. These stakeholders work on the back end of the e-procurement application and as a consequence they have a different view of e-procedures. (See chapter 18 for a detailed discussion of the underlying complexity of eliciting and defining stakeholders' requirements for e-Government applications). Indeed, some complex administrative procedures will ultimately require the processing of pieces of information by many stakeholders, who work for different agencies and departments; quite often, only a few stakeholders have the entire view of the underlying workflow. In this context, it seems extremely important to provide each participant involved in the development process with multiple views for e-procedures:

- an overall view of the different steps in the underlying workflow process

- a detailed view of tasks that users should perform at every step of the procedure

- a detailed view of how the system supports user tasks in the execution of a procedure

This chapter shows how to employ abstract representation (called models) to supporting these three views of e-procurement applications. Such models can be used to communicate design options and design decisions to all participants of the development process, including the stakeholders. Beyond that, models are the ideal place for stimulating information exchanges, for sharing them and for recording them. The approach presented in this chapter combines several notations for representing 
models that can additionally be used for engineering interactive systems. Our main goal is twofold:

- define in an unambiguous way the behavior of several components (mainly user tasks and e-procurement application behavior)

- provide a means to ensure the cross-consistency between these two complementary views of the same socio-technical system

For this very purpose, we use two different notations for describing two types of models: tasks models that represent the actions users should perform at every step of an administrative procedure, and system models that describe how design options can be implemented by the system to support user tasks. The word "system" is used here to refer to the interactive and functional part of the e-procurement application. It does not encompass the hardware and network parts even though they could be modeled with adequate notations. We illustrate user task modeling by using a hierarchical notation called HAMSTERS (which stands for Human-centered Assessment and Modeling to Support Task Engineering for Resilient Systems). The system behavior is described by the means of the StateWebCharts notation dedicated to the modeling of Web-based applications. The use of these notations is illustrated on a real case study extracted from the French Regional Administration (Région Midi-Pyrénées). When used in an integrated and complementary way, these models can provide the various stakeholders with detailed and structured information to understand the interrelations between individual user's tasks and the system's behavior. [H1]Development process for e-procurement applications Models are valuable tools for reducing ambiguities of specifications, making large and complex projects more manageable, documenting the design, and supporting the communication among developers and stakeholders. Models can be useful at a 
specific phase of development as well as throughout the application life cycle. The main goal of this chapter is to show how model-based approaches can contribute to the development process of e-procurement applications. To understand this, Figure 19.1 depicts a life cycle that illustrates the use of models along the development process of Web applications as proposed by Scapin, Vanderdonckt, Farenc, Bastide, Bastien, Leulier, Mariage \& Palanque (2000). Currently, there is no consensus on which phases of development are required or which life cycle better describes the development process of e-procurement applications. Nonetheless, the lifecycle for Web development can be helpful in understanding how e-procurement applications are developed to be deployed on the Web platform. As we shall see, the lifecycle presented in Figure 19.1 is an iterative process made up of six steps:

1. Requirements engineering: Identify the main goals of the stakeholders, context of use, and requirements (see Chapter 18).

2. Specification: Produce models for describing the context of use and requirements gathered in the previous phase. Detailed models formalize requirements including user tasks and e-procurement application behavior, for instance.

3. Design: Refine the specifications according to their content. At the end of this phase a navigation map and page templates are prepared. This phase produces detailed specification to guide the implementation of the Web application.

4. Development: Construct the Web application, produce the Web pages, and integrate tools for visualizing media (e.g., sound, video). At the end of this phase, all the pages have content, links and graphic elements incorporated: the application is delivered. 
5. Site usage and evaluation: Evaluate advanced prototypes with end users. The product of previous phases is checked with respect to the requirements and the context identified in the first phase. For further information about usability evaluation along the development process the interested reader should refer to chapter 17.

6. Maintenance: Gather new information, and plan modifications that have been requested from the use and evaluation phase.

\section{*** INSERT Figure $19.1 * * *$}

[Caption]Figure 19.1 Life Cycle for Web application development

The process of Figure 19.1 is cyclical going sequentially through the 6 phases presented above. However, it is widely known that interactive applications development requires faster and sometimes incomplete iterations especially when prototyping activities have to be performed. Fast iterations are represented in the model by the two arrows (in dotted lines) in the middle of the loop. In Figure 19.1, the arrow on the left-hand side indicates possible shortcuts of the specification phase. Indeed, at the beginning of design, the information architects and web designers may start immediately to design the site, to have precise information to exchange and discuss with the stakeholders. The arrow on the right-hand side (implementation changes) represents a possible shortcut for increasing development speed and taking into account in a more central manner the usage and the evaluations.

In the next section we will focus on models used during the specification phase of administrative e-procurement.

[H1]Modeling of e-procurement applications An e-procurement application should coordinate seamlessly the relationships between information concerning the organization, the underlying workflow process, and the 
database (see Pontico, Farenc \& Winckler, 2006). Figure 19.2 presents a graphical representation of such information exchange between these three components in the case of an e-procurement application dealing with the submission and evaluation of students' requests for scholarships. This example is similar to many of currently available e-procurements in terms of coordination of activities, responsibilities and resources. This example does not exhibit the critical aspects of the administrative procedures and of the data handled in such contexts, but it has the advantage of both simplicity and of conveying most of the concepts we want to address in this chapter. *** INSERT Figure19.2***

[Caption] Figure19.2 Information flow between the organization, the process, and the database

As we can see in Figure 19.2, a user can evaluate a submission only if the corresponding document is available in the database and if the organization grants him or her the rights associated to the role of reviewer. Notice that the task "Evaluate a submission" is represented only through the relationships between actors. This way of representing user tasks can be easily justified from an information systems point of view, but it raises a big problem for understanding user activity on the system, especially when actors must cooperate to perform a single activity (e.g., support the discussion of a student application among administrative clerk and possibly a school principal). Such graphical representation is usually perceived by the developers of data intensive web application as sufficient (Ceri, Fraternali, Bongio, Brambilla, Comai \& Matera 2003) as it supports quite efficiently the identification of information that has to be stored in the database. However, when it comes to the design of the interactive part of the e-procurement application, there is a need to provide more accurate behavioral description. In order to do so, two complementary perspectives 
have to be described: all the tasks a user can perform with the system (this representation is called tasks model), and a specification of all function- and scheduling-centered behaviors that should be embedded into administrative eprocurement applications (this description is called the system model). These two models including the information they embed and how they are built are described in the following sections.

[H2] Modeling user tasks

Task modeling has been proposed as a mean of recording information gathered in the task analysis phase and is widely recognized as one fundamental way to focus on the specific user needs and improve the general understanding of how users may interact with a user interface to accomplish a given interactive goal (Diaper \& Stanton, 2004). Task models do not imply any specific implementation, so that one can focus on dependencies between activities, availability of resources required to perform tasks and steps users should follow to achieve a task.

Most notations for describing tasks feature a hierarchical organization of goals that are connected by logical and temporal operators for expressing dependencies between them. A task model should describe what users must do to accomplish a given goal without including how the system processes information even though this might determine the outcomes of a task execution. This is usually represented by alternative paths in the task model making explicit the possible outcomes. For this reason, many scenarios (which describe a unique sequence of task execution) can be produced from a single task model. As we shall see in the case study below, one of the advantages of modeling user tasks and scenarios is they help to analyze conflicts between users and administrative goals long before system constraints are considered.

[H2] Modeling the system 
Several user interface (UI) description languages (UIDLs) exist for describing the user interface and the expected system behavior (see Shaer, Jacob, Green \& Luyten 2009). A UIDL might cover one or more of three different aspects of the UI: the static structure of the user interfaces (including the description of user interface elements i.e., widgets - and their composition), the dynamic behavior (the dialog part, describing the dynamic relationships between components, including event, actions, and behavioral constraints), and the presentation attributes. For the sake of simplicity, we will focus only on the behavioral aspects of the systems and, in particular, how we can represent all the user navigation available on a Web portal featuring eprocurement applications. This exhaustive set of navigations is called the system model and should provide a clear description of system behavior, including how the system processes user inputs and generates appropriate output. This system model must then be exploited for prototyping the UI and in the implementation phases of the development process.

[H1]Case study: modeling e-procurement applications

To illustrate the complementary use of task and system models, this section presents a case study ${ }^{1}$ of an e-procurement application provided by the Regional French Administration Midi-Pyrénées (RMP, www.midipyrenees.fr). We introduce all actors involved and their interactions along the administrative procedures; however, due to space constraints the models only embed citizens' interactions.

[H2] Informal description of the case study

Our case study concerns an e-procedure developed as part of the BRPE program (the French acronym for "Regional Scholarship for First Equipment") whose aim is to provide students with scholarships for buying the required equipment (e.g., for

\footnotetext{
${ }^{1}$ This case study omits some internal aspects of the application.
} 
hospitality students the purchase of knives, aprons, and suits) for attending classes in vocational high schools. Like many other governmental programs, BRPE is a complex program that integrates actors with diverse juridical status such as citizens (students/parents), units of the regional governmental (RMP) units, state governmental units (the accounting department), and educational units (high schools). Educational units are controlled by Education Offices, which negotiate once a year BRPE scholarships entire budget with RMP. For the sake of simplicity, Education Offices, accounting departments and national banks will be considered as "state units".

A student can apply for a BRPE scholarship only once, and only while attending a specific technical program in a vocational high school. High-school principals are in charge of advising students about the calendar and procedures and helping them prepare applications. BRPE applicants obtain forms from high-school principals. For students under the age of majority, their parents or legal guardian must sign the form. They send the forms and required documents (e.g., a bank account statement) to the high-school principals, who verify the completeness of the forms and send the complete ones to RMP. On receipt, RMP agents analyze BRPE applications. If the application is accepted by RMP, the accounting department (a state institution distinct from RMP) pays the BRPE scholarship through bank transfer to the account of the student (or his or her parents). Figure 19.3 shows the general procedure and depicts how the BRPE processes (gray boxes) are connected to outside processes (black boxes).

*** insert Figure $19.3 * * *$

[Caption] Figure19.3 Overview of the BRPE process 
From an administrative point of view, the procedure starts with the annual definition of the amount of money allocated per scholarship which varies according to the technical program (Figure 19.3, step 1). Scholarships are subject to annual budget approval from the RMP's council (step 2) which determines the number of scholarships that can be founded. Students do not send applications directly to RMP: the process is mediated by the principals, who notifies students (step 4) and explains how they should fill in the form (step 5). Principals are also responsible for checking that all required documents are present and that student regularly attend a vocational high school (step 6). RMP receives student applications and verifies again their correctness and eligibility (step 8). Problems (e.g., fraud, missing information) are reported to the principals (step 7), who can also monitor (step 6) the status of applications of students attending a program at their schools. Eligible applications are duly recorded, and letters of credit are sent to recipients (step 9). Finally, RMP addresses a payment request (step 10) to the accounting department (step 11). Table 19.1 shows the roles that have been identified for the application — "student" and "Administrative clerks" — and their corresponding (allowed) tasks. The role "student" includes profiles "students without login" and "registered students". The task "Query (for scholarships)" is available to everyone, but a user can apply for scholarship only if s/he is logged in the system. The user role "Administrative clerks" refers to someone who is responsible for supervising the submissions.

Table 19.1. Tasks associated with BRPE scholarship applications

\begin{tabular}{l|c|c|l}
\hline Role & \multicolumn{1}{|c|}{ User Profile } & Pre-conditions & \multicolumn{1}{c}{ (allowed) Tasks } \\
\hline Student & Student without login & none & $\begin{array}{l}\text { Query (for scholarships) } \\
\text { Create an account } \\
\text { Log into the system }\end{array}$ \\
& Registered students & logged in & $\begin{array}{l}\text { Query (for scholarship) } \\
\text { Update user account } \\
\text { Apply for scholarships } \\
\text { Monitor status of requests }\end{array}$ \\
\hline
\end{tabular}




\begin{tabular}{l|c|l|l}
\hline $\begin{array}{l}\text { Administrative } \\
\text { clerks }\end{array}$ & $\begin{array}{c}\text { Full control over } \\
\text { scholarship } \\
\text { applications }\end{array}$ & logged in & Process scholarship request \\
Notify students
\end{tabular}

[H2] Modeling user tasks for BRPE

User tasks for the BRPE have been modeled using HAMSTERS which is a graphical notation for describing task models hierarchically. The notation is supported by a software tool for the editing and simulation of the models and is publicly available ${ }^{2}$. The elements of task models described by HAMSTERS include various tasks types (e.g. abstract, system, user, and interactive tasks) aimed at expressing who performs the task. Similar to other task model notations such as the Concur Task Tree (CTT) notation (Paternò, Mancini \& Meniconi, 1997), temporal and logical operators (e.g. "[]" for choice, ">>" for sequence, “|||" order independency) are used to define the relationships between tasks. Figure 19.4 presents a task model describing the set of actions and their temporal ordering in order for a user to log into the system (user goal represented at the top level of the task model). In order to reach this goal the user has to perform the abstract task "Provide identification" which, in sequence (operator $>>$ ) will be processed by the system "Validate user id". In order to perform the identification task the user can perform in any order (operator || $\mid$ ) "provide email" and “provide password" tasks.

*** Insert Figure $19.4 * * *$

[Caption] Figure 19.4 Task model in HAMSTERS, describing a simple user login. Figure 19.5 shows the task model of the students represented using HAMSTERS notation corresponding to the goal "Submit BRPE". The level immediately below describes the tasks that users can perform without having a user account, such as "Query (for scholarships)", "Create account" and "Select scholarship" in any order

\footnotetext{
${ }^{2}$ htpp://www.irit.fr/ICS/hamsters
} 
(operator $\|||$ ). Connected to that operator, the sequence operator " $>>$ " indicates the fact that the user must perform in sequence, first the task "Log into the system" and then the task "Manage account". The decision to start this sequence is performed in any order with the tasks of the same level. The task "Select scholarship" is a cognitive task which refers to user's inner decision process. The process of refinement of tasks proceeds until all the details for understanding the user goals and activities have been reached. For example, the task "Query (for scholarships)" is decomposed into "provide keyword" and "show results" (which have to be performed in sequence ("“>”)), representing respectively the expected user input and the outcome provided by the system.

The task 'Apply for scholarship' encompasses a set of subtasks that are required to accomplishing the procedure that follows Figure 19.5 (Administrative constraints cause the administration to request paper-based certificates, so the subtask "provide certificates" is not supported by the system). Similarly, the task model describes how a student can "submit forms" either online on its printed version (represented by the choice operator "[]").

*** insert figure $19.5 * * *$

[Caption] Figure19.5 HAMSTERS task model for the application BRPE Exploiting the task model presented in Figure 19.5 can yield many scenarios. Table 19.2 shows three possible scenarios for the task "Apply for scholarship". Scenario 1 considers the situation of a student that connects to the system and ultimately submits forms online. In scenario 2 the user also connects to the system but finally decides to submit printed forms. Scenario 3 describes the situation where a user updates account information after the application is submitted. This might violate the constraint of applying for a scholarship only while attending a specific technical program in a 
vocational high school. Notice that these scenarios do not impose any particular implementation; user tasks can be better understood without having information about how the system will support them. This kind of analysis is possible because it considers user tasks from the perspective of the users' needs from the application, rather than how to represent the user activity using a particular system.

Table 19.2. Three scenarios related to "manage account" subtask

\begin{tabular}{|l|l|l|}
\hline Scenario 1 & Scenario 2 & Scenario 3 \\
\hline Apply to for scholarship & Apply to for scholarship & Apply to for scholarship \\
Prepare documents & Prepare documents & Prepare documents \\
Request application & Request application form & Request application \\
form & Fill in forms & form \\
Fill in forms & Submit forms & Fill in forms \\
Submit forms & Submit printed forms & Submit forms \\
Submit forms online & Provide certificates & Submit forms online \\
Provide certificates & Process request & Provide certificates \\
Process request & Monitor status & Process request \\
Monitor status & & Update account \\
& \multicolumn{2}{|l}{ Update school } \\
\hline
\end{tabular}

[H2] Modeling the navigation in the BRPE systems

To describe the navigation of the BPRE application, we employ the StateWebCharts notation (SWC) (Winckler, Barboni, Farenc, \& Palanque, 2004). SWC is a formal description technique based on Harel's (1987) StateCharts and developed to specify the dynamic behavior of Web applications. StateCharts can be defined as a set of states, transitions, events, conditions, variables, and their interrelationships. The behavior described in SWC is directly related to the user interface. Sates in SWC, are depicted on the user interface by means of containers for objects (graphic or executable objects) e.g. HTML pages. During the execution of the model the current state (and its content) is made visible to the users. SWC transitions explicitly represent how user events trigger state changes in a model (user actions are graphically represented as continuous arrows). Autonomous behaviors are graphically 
represented as dashed arrows. When a user selects a transition the system leaves the current state, which becomes inactive, letting the target state be the next active state in the configuration. Figure 19.6 presents an excerpt of a system model using SWC supporting user login.

*** Insert Figure 19.6***

[Caption] Figure 19.6 Simple login described using SWC

In Figure 19.6 the state "login" contains three static states "fill in email and pwd", "logged in", "error: try again" that describe the three possible pages the user can see whilst navigating the application. The state "check password" is a transient state that represents the information processing performed at the server side without any visual representation to the user. The round-shaped state is an "end state" used to describe the end of the execution of the application. Notice the SWC model presented in Figure 19.5 is just one of many alternatives for defining the system behavior for performing the task login depicted in Figure 19.4; nonetheless, it describes one agreed upon design for the navigation of the login part of the BRPE application.

Figure 19.7 shows the complete navigation model for BRPE system including the support for the tasks "Log into the system" and "Query (for scholarships)". In addition to the functions required to support the tasks represented in the task model, it features some transitions supporting content-based navigation such as "home", “connected". Simply by connecting states by means of transitions we can create all the navigation required by the users, whether it concerns content-based navigation or is navigation required to follow a specific procedure.

*** insert Figure19.7 ***

[Caption]Figure 19.7 SWC modeling for BRPE e-procurement application [H2]Prototyping e-procurement applications from specifications 
SWC notation is supported by a computer tool suite making it possible to edit and execute models. This specificity support iterative prototyping as described in the development process of Figure 19.1. After we have created and verified that the navigation model embed our requirements, we can create the Web pages for the eprocurement application that corresponds to the SWC model. When the navigation is described, the graphical part of the user interface can be connected to the states of the model while user events can be connected to transitions. Executing the models thus allow for simulation of the user interface according to the high fidelity prototyping philosophy. The SWCEditor supports the simultaneous simulation of SWC models and the execution of the corresponding Web pages. Figure 19.8 provides a view-at-aglance of this process. The navigation modeling for the digital library of BRPE is presented on the left-hand side of Figure 19.8, highlighting the current state in the simulation (i.e., the state "home"). Its right-hand side presents the corresponding implementation of the home page. We also can observe in the center of Figure 19.8 a dialog window showing a list of transitions going out from the current state "home". This list represents the set of links currently available for the user navigation. $* * *$ insert figure $19.8 * * *$

[Caption]Figure 19.8 Prototyping BRPE using SWC models connected to a web page [H1] Discussion

This chapter has illustrated the use of task models and system models for dealing with the development of e-procurement applications. The case study presented might look small but it exhibits the huge importance that models will play in the development of even more complex applications. Without appropriate support from models, the development team will get lost and the chance of unexpected and undesired behaviors would increase. These models provide different point of views on the same socio- 
technical system so that when working in multidisciplinary teams all stakeholders can use the most suitable model to make explicit their concerns and requirements to be embedded in the final application.

Most of existing development methods for web applications bases their conceptual modeling on the objects (or data) and their related methods, functions, or services, and they derive tasks from the traditional CRUD (Create, Read, Update, Delete) pattern: tasks are limited to basic operations on objects and their relationships (Ceri et al, 2003). These data-centric development methods only integrate the graphical and interaction designers' point of view by means of stereotypes preventing any creativity about the user interface presentation and navigation.

The use of task models reinforces the focus on users during the development process of application. Indeed, we must pay attention to the users' tasks to help users navigate the application effectively and efficiently. Several other approach for designing eprocedures exist, many of which do not include explicit representation of user tasks. Even though task modeling is widely considered as helpful activity that lets design analyze the user activity without the influence of technological constraints, the actual use of task models for the design of e-procurement applications is often misunderstood, mainly because current approaches for the design do not provide any guidance on how to integrate task models into the design process. This chapter focused on tasks models and system models rather than on the underlying workflow process of e-procedure applications. Nonetheless, the workflow can be derived from the co-execution of task models and navigation models.

One of the key contributions of the models presented in this chapter is that task models (HAMSTERS) and system models (SWC) can be integrated and that their compatibility can be assessed prior to implementation. For instance, if the task model 
features 4 interactive tasks then the system model should embed the same set of transitions between states. Beyond that lexical compatibility, syntactic compatibility has to be insured too. For instance, if there is a sequence of actions in the task model (describing a constraint in user activity that can be administrative, physical or cognitive) this constraint must be reflected in the system model. This is precisely where the power of models is exacerbated. While checking-by-testing such compatibility is impossible due to the potentially very large number of states and state changes, models bissimulation and model-checking techniques can provide efficient solutions. Bissimulation of task models and system models is detailed by Barboni, Ladry, Navarre, Palanque \& Winckler (2010).

One of the key assumptions of the approach presented here is that user activity should be represented in tasks models only and that system behavior should only be represented in the system models. Keeping the content of task models different from (but compatible with) the system models allows provide methodological guidelines about the construction of these models. A task model should not inform how many pages a user must visit to accomplish a task because this is often a system constraint. A scholarship application form such as BRPE might feature a single page in a Web browser on a desktop but designers can decide to slice the form in several pages accordingly to groups of information requested. Moreover, accessing the same application through a mobile Web browser will request that form will be sliced into many pages. Notwithstanding the number of pages used to present the BRPE form, the user task remains the same.

It is important to note that several other models have been proposed over the last decade that might fit with the objectives and processes presented in this chapter. We 
only presented SWC and HAMSTERS to illustrate the applicability of the concepts and the benefits that can be gained following such an approach.

[H1] Success factors for modeling users and systems behaviors for e-procurement applications

The following check list is supposed to help the reader with key information while using a model-based approach and a model-based development process as presented in this chapter.

- Keep clear the distinction between the information included in the task model and the system model

- The system model should exhibit infinite sequences supporting the same tasks to be executed by several users one after the other. The execution of one task should not impact the future execution.

- The tasks model should terminate. An iterative tasks model would represent the fact that infinite sequences of action are required for reaching a goal.

- Do not start using models if there is no tool support for editing the models

- A simulation engine allowing to "see" how the model behaves is required

- Make sure to reflect in the models all the changes that are made on the application otherwise there would lose their interest as a tool for analysis and information sharing

- Make sure that your models are readable and understandable by the various stakeholders so that they can validate and modify them

- $\quad$ Reading and building a model requires different skills (don't expect stakeholder with a non-technical background to build system models) 
- If you use a multiple models approach make sure that there is a support to assess cross-models compatibility as performing it by hand is usually unmanageable

- Do not try to use models everywhere. Models should be kept for the complex part of the system and for the complex user tasks. The rest of the system can be built using prototyping approaches. There are different ways to identify these complexities (looking at user and/or system failures are a good indicator).

[H2]References

Barboni, E., Ladry, J.-F., Navarre, D., Palanque, P., \& Winckler, M. (2010). Beyond Modelling: An Integrated Environment Supporting Co-Execution of Tasks and Systems Models. In proceedings of ACM Symposium on Engineering Interactive Systems (EICS'2010), New York, United States : Sheridan.

Ceri, S., Fraternali, P., Bongio, A., Brambilla, M., Comai, S. \& Matera, M. (2003).

Designing Data-Intensive Web Applications. San Franscico, United States: MorganKaufmann Publishers.

Diaper, D., \& Stanton, N. A. (2004). The Handbook of Task Analysis for HumanComputer Interaction. Mahwah, New Jersey: Lawrence Erlbaum Associates.

Harel, D. (1987). StateCharts: A Visual Formalism for Complex Systems. Science of Computer Programming, 8(3), pp. 231-274, Amsterdam: Elsevier.

Martinie, C., Palanque, P., \& Winckler, M. (2011). Structuring and Composition Mechanism to Address Scalability Issues in Task Models. In Proceedings of IFIP TC13 Conference on Human-Computer Interaction (INTERACT 2011), 593-611, LNCS 6949, Berlin: Springer. 
Paterno, F., Mancini, C., \& Meniconi, S. (1997). ConcurTaskTrees: A Diagrammatic Notation for Specifying Task Models. In Proceedings of the IFIP TC13 Conference on Human-Computer Interaction (INTERACT'97), 362-369, London: Chapman \& Hall. Pontico, F., Farenc, \& C., Winckler, M. (2006). Model-based support for specifying eService eGovernment Applications. In Proceedings of the $5^{\text {th }}$ International Workshop on TAsk MOdels and DIAgrams (TAMODIA'2006), 54-67, LNCS 4385.

Berlin: Springer.

Scapin, D., Vanderdonckt, J., Farenc, C., Bastide, R., Bastien, C., Leulier, C., Mariage, C., \& Palanque, P. (2000). Transferring Knowledge of User Interfaces Guidelines to the Web. In Proceedings of TWWG 2000: International Conference on Tools for Working With Guidelines, 293-304, London, UK: Springer.

Shaer, O., Jacob, R. J. K;, Green, M., \& Luyten, K. (special editors). (2009). User

Interface Description Languages for Next Generation User Interfaces. Special Issue of ACM Transactions on Computer-Human Interaction (ACM TOCHI), 16(4), New York: ACM.

Winckler, M., Barboni, E., Farenc, C., \& Palanque, P. (2004). SWCEditor: a ModelBased Tool for Interactive Modelling of Web Navigation. In Proceedings of International Conference on Computer-Aided Design of User Interface (CADUI'2004), Kluwer Academic Publisher. 